\title{
Plasminogen Activator Inhibitor-1 in Patients with Kawasaki Disease: Diagnostic Value for the Prediction of Coronary Artery Lesion and Implication for a New Mode of Therapy
}

\author{
HIDEAKI SENZAKI, TOSHIKI KOBAYASHI, HIRONORI NAGASAKA, HIROFUMI NAKANO, \\ SHUNEI KYO, YUJI YOKOTE, AND NOZOMU SASAKID \\ Department of Pediatric Cardiology and Pediatrics, Saitama Heart Institute, Saitama Medical School \\ Hospital, Saitama, 350 Japan
}

\begin{abstract}
Kawasaki disease (KD) in children takes the form of acute systemic vasculitis, which causes coronary artery dilation and aneurysm formation in $10 \%$ to $15 \%$ of the patients. We have recently shown that matrix metalloproteinases (MMPs) are intimately involved in coronary arterial wall destruction and the resultant formation of coronary artery lesions (CALs) in this disease. Plasminogen activators (PAs) are known to be a major pathway of MMP activation, and this suggests that their inhibitor, plasminogen activator inhibitor-1 (PAI-1), also plays important roles in the development of CALs in KD. The present study was conducted to test the hypothesis that circulating levels of PAI-I are related to CAL formation in KD. Plasma levels of PAI-1 were measured by enzyme-linked immunoassay in $37 \mathrm{KD}$ patients without CALs (group 1) and $7 \mathrm{KD}$ patients with CALs (group 2). Blood samples were obtained before and after i.v. gammaglobulin therapy (IVGG), and in the convalescent stage. Levels of PAI-1 were significantly higher in KD patients before IVGG than in 18 age-matched healthy control subjects $(p<$ 0.01 ). More importantly, both pre-IVGG and post-IVGG levels of PAI-1 were significantly higher in group 2 than in group 1 ( $p$ $<0.01)$. Furthermore, PAI-1 levels of 9 patients from group 1
\end{abstract}

\section{ABSTRACT}

who showed pre-IVGG PAI-1 levels higher than the minimum PAI-1 level in group 2 significantly decreased after IVGG, whereas PAI-1 levels of group 2 patients remained persistently elevated, further suggesting a close association between PAI-1 and CAL development in KD. Thus, PAI-1 may be useful as a predictive marker for CAL development in KD. Studies of the effects of PA inhibition on coronary outcome may provide evidence that PA is a viable therapeutic target for the prevention of KD-related CALs. (Pediatr Res 53: 983-988, 2003)

$\quad$ Abbreviations
MMP, matrix metalloproteinase
PA, plasminogen activator
PAI-1, plasminogen activator inhibitor-1
KD, Kawasaki disease
CAL, coronary artery lesion
IVGG, i.v. administration of gammaglobulin
TIMP, tissue inhibitor of matrix metalloproteinase
TNF, tumor necrosis factor
IL, interleukin

The extracellular matrix of the arterial wall provides a strong structural safety net that resists arterial expansion in a healthy individual. Arterial aneurysm development is thought to be the consequence of degradation of these structural proteins by proteolytic enzymes derived mainly from inflammatory cells infiltrating into media and adventitia (1-3). Matrix metalloproteinases (MMPs) have been implicated in this degradation

Received July 2, 2002; accepted December 18, 2002.

Correspondence: Hideaki Senzaki, M.D., Department of Pediatric Cardiology, Saitama Heart Institute, Saitama Medical School Hospital, 38 Morohongo, Moroyama, Saitama 350, Japan; e-mail: hsenzaki@saitama-med.ac.jp

This study was supported by a grant from Kawano Memorial Foundation (No. 10-3) (H.S.) and a Medical Research Grant from Nipro Corporation (H.S.).

DOI: 10.1203/01.PDR.0000061566.63383.F4 process, and are thought to play dominant roles in the formation of abdominal aortic aneurysms (3-6).

Plasminogen activators (PAs) and plasmin (a serine protease) are known to be activated in response to several inflammatory conditions and to act as triggers activating the MMP pathway (7, 8). In addition, recent study of aortic aneurysm in a rat model has demonstrated that blocking PA activity via local overexpression of plasminogen activator inhibitor-1 (PAI-1) prevents formation of arterial aneurysms by inhibiting MMP activation, suggesting a critical role for PA-mediated MMP activation in arterial wall destruction and resultant aneurysm formation $(6,9)$.

Kawasaki disease (KD) in children takes the form of acute systemic vasculitis, which causes coronary artery dilation and aneurysm formation in $10 \%$ to $15 \%$ of patients during its acute 
stage $(10,11)$. We have recently shown that, in KD, MMPs may also be intimately involved in coronary arterial wall destruction and the resultant formation of coronary aneurysms (12). Given the inhibitory effects of PAI-1 on MMP activation and our previous data indicating important roles for MMP in the formation of coronary artery lesions (CALs) in KD, it is possible that PAI-1 is also involved in the development of CALs in KD. The present study was conducted to test the hypothesis that circulating levels of PAI-I are related to CAL formation in $\mathrm{KD}$.

\section{METHODS}

Forty-four consecutive KD patients were prospectively recruited. These subjects were subdivided into 2 groups: 37 patients without CALs (group 1), and 7 patients with CALs (group 2). The blood samples were obtained between 9 and 10 am by nontraumatic needle aspiration from the antecubital vein. Immediately after samples were centrifuged, plasma was cooled to $-80^{\circ} \mathrm{C}$ and stored at that temperature until assays were performed. Plasma levels of PAI-1 were measured by enzyme-linked immunoassay (ELISA) using MAb (13). Because endogenous PAs (mostly tissue PA) are saturated by PAI-1 and circulate predominantly in complex with PAI-1 (14), the PA/PAI-1 complex level reflects the level of PA (14). Therefore, we estimated the level of PA from PA/PAI-1 complex levels measured by ELISA.

All KD patients fulfilled the revised $1984 \mathrm{KD}$ criteria of the Kawasaki Disease Research Committee of Japan (15). Coronary arteries with diameters of $4 \mathrm{~mm}$ or greater were considered to exhibit CALs (15). All KD patients were treated with i.v. administration of gammaglobulin (IVGG, $400 \mathrm{mg} / \mathrm{kg} / \mathrm{d}$ for 5 consecutive days) combined with oral aspirin $(50 \mathrm{mg} / \mathrm{kg} / \mathrm{d})$. Data were obtained before and after gammaglobulin treatment, and near the time of hospital discharge (convalescent stage). These data were compared with those from 18 age-matched afebrile control subjects (group 3). Group 3 included patients who had undergone reparative surgery for total anomalous pulmonary venous connection, small subpulmonary ventricular septal defect, or atrial septal defect more than $1 \mathrm{y}$ before this study. None of the afebrile controls were receiving medication, and the results of their echocardiogram, ECG, laboratory tests and physical examination were all normal. Written informed consent was obtained from the parents of all patients, and the procedures were approved by The Saitama Medical School Committee on Clinical Investigation.

\section{Statistical analysis}

Data are presented as mean \pm SEM. Comparisons among groups were made by ANOVA followed by Bonferoni correction. Differences in PAI-1 and PA/PAI-1 complex levels between time points were tested by repeated measures of ANOVA. $p<0.05$ was considered to indicate statistical significance.

\section{RESULTS}

Table 1 summarizes patient characteristics of each group. The term "days of illness" indicates the points during the time course of illness at which blood samples were obtained, and there was no significant difference in these time points between groups 1 and $2(p=N S)$. We defined onset of illness as the day on which the patient developed fever. The only significant difference in data between groups 1 and 2 was that group 2 had a higher C-reactive protein level than group 1 after IVGG therapy. None of the group 2 patients had CAL either before IVGG or before the second samples were taken. The average time at which CALs developed was $13.9 \pm 1.7 \mathrm{~d}$ of illness.

Figs. $1 A$ and $B$ demonstrate circulating levels of PAI-1 and PA/PAI-1 complex in the afebrile control group and in KD patients before IVGG therapy. The values obtained for the afebrile control group (PAI-1, $19.3 \pm 2.5 \mathrm{ng} / \mathrm{mL}$; PA/PAI-1 complex, $5.1 \pm 0.1$ ) were quite similar to those previously reported for healthy adults (16). Therefore, although group 3 subjects were not truly healthy subjects, they can be considered comparable to healthy children for the purposes of the present study. The levels of PAI-1 and PA/PAI-1 complex in KD patients were significantly elevated compared with those of group $3(p<0.01)$. More importantly, the PAI-1 level of group 2 patients was significantly higher than that of group 1 (150 \pm 21 versus $77 \pm 10 \mathrm{ng} / \mathrm{mL}, p<0.01)$, whereas groups 1 and 2 had similar PA/PAI-1 complex levels, suggesting that KD patients with CALs have an imbalance between plasminogen activation and inhibition at the very early stage of illness. To further elucidate the balance of this fibrinolytic system, we compared the ratio of PA/PAI-1 complex to PAI-1 among the 3 groups (Fig. 1C). This ratio was significantly lower in group $2(0.11 \pm 0.02)$ than in groups $1(0.24 \pm 0.03)$ and $3(0.30 \pm$ $0.04)$. The ratio was lower in group 1 than in the controls, but the difference was not statistically significant.

Figure 2 shows the serial changes in PAI-1, PA/PAI-1 complex and the ratio of PA/PAI-1 to PAI-1 in KD patients. After IVGG, levels of PAI-1 remained elevated in both KD

Table 1. Patient characteristics

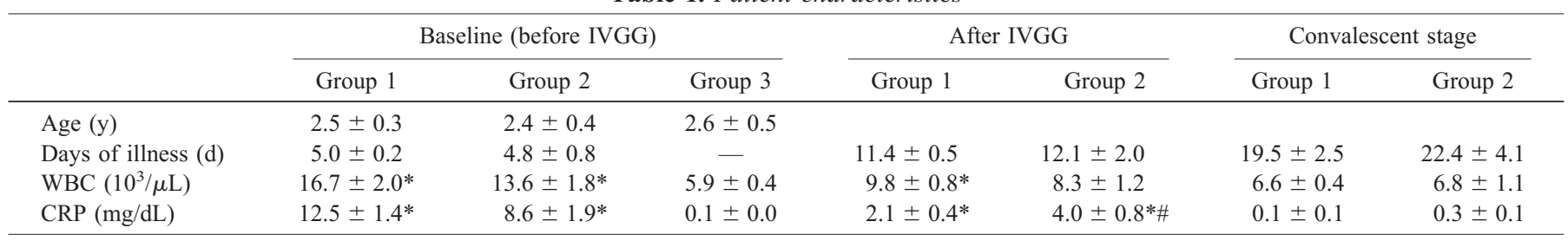

IVGG, intravenous gamma globulin; WBC, white blood cell count; CRP, C-reactive protein. $* p<0.05 v s$. afebrile controls (group 3), $\# p<0.05 v s$. group 1 by unpaired $t$-test. 
A

PAI-1
B
PA/PAI-1 Complex
PA/PAI-1 Complex
C

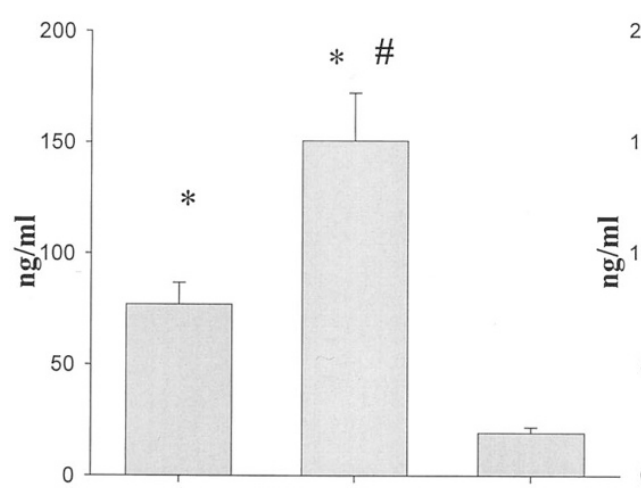

Group 1 Group 2 Group 3

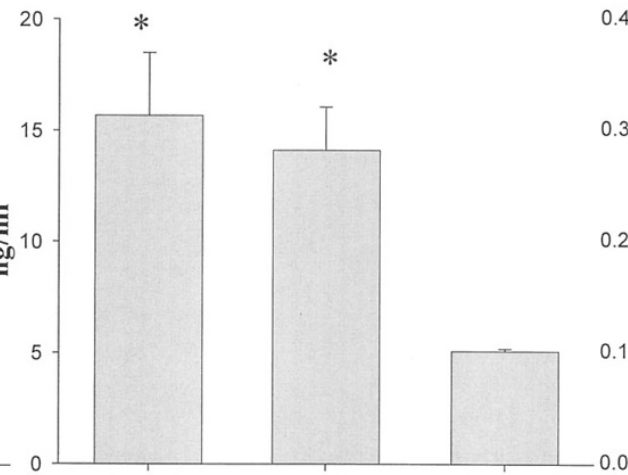

Group 1 Group 2 Group 3

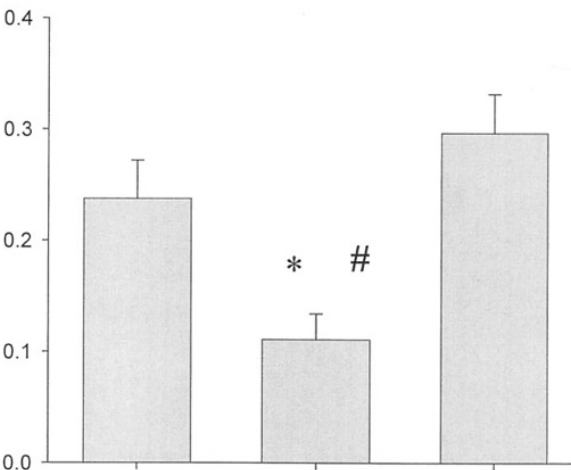

Group 1 Group 2 Group 3

Figure 1. Circulating levels of PAI-1 $(A)$, PA/PAI-1 complex $(B)$, and the ratio of PA/PAI-1 complex to PAI-1 $(C)$ for each group before i.v. gammaglobulin therapy. ${ }^{*} p<0.05$ vs. group $3, \# p<0.05$ vs. group 1.

A

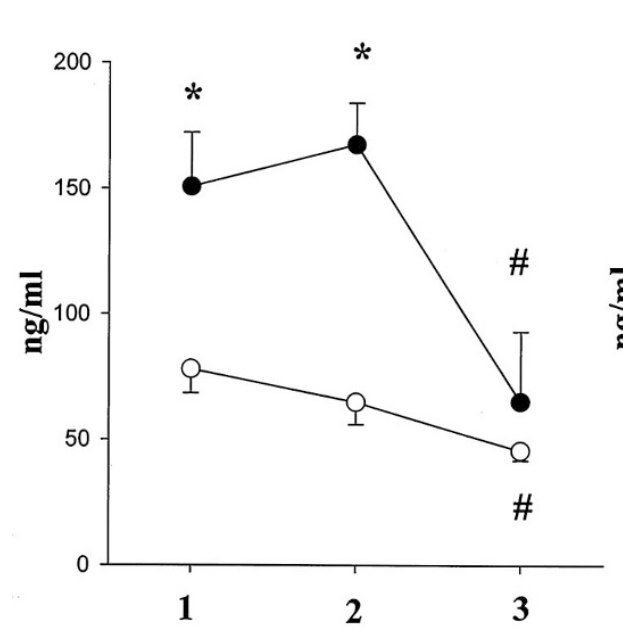

B PA/PAI-1 Complex

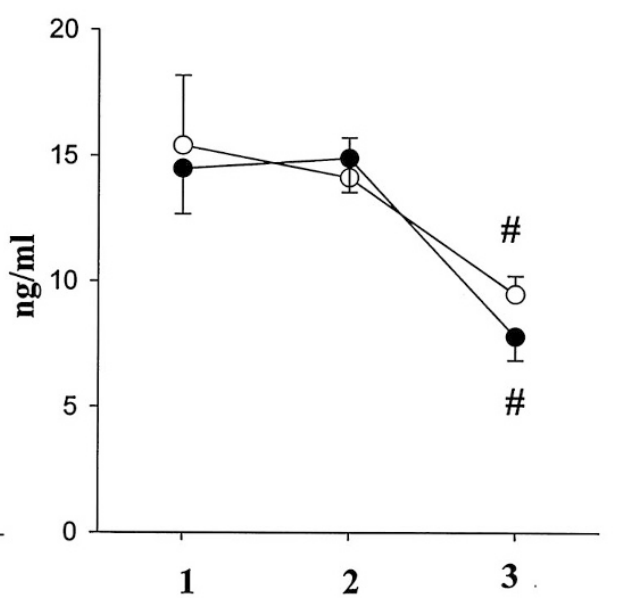

C PA/PAI-1 Complex vs. PAI-1 Ratio

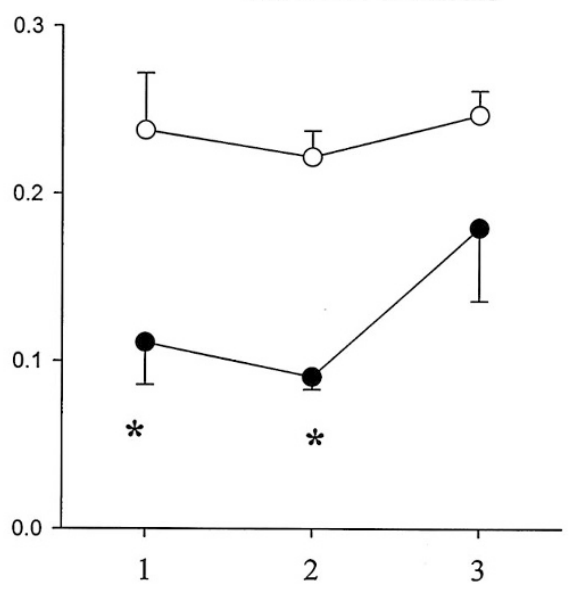

Figure 2. Serial changes in PAI-1 $(A)$, PA/PAI-1 complex $(B)$, and the ratio of PA/PAI-1 complex to PAI-1 $(C)$ in Kawasaki disease patients. $\bigcirc$, group 1; group 2; 1, before IVGG; 2 , after IVGG; 3 , converlescent stage; ${ }^{*} p<0.05 v s$. group 1 ; $\# p<0.05 v s$. before IVGG.

groups, and PAI-1 was significantly higher in group 2 than in group $1(168 \pm 16$ versus $65 \pm 9 \mathrm{ng} / \mathrm{mL}, p<0.01)$. In the convalescent stage, PAI-1 levels of the $2 \mathrm{KD}$ groups fell to similar levels $(p=N S)$, but those levels were still significantly higher than those of group 3. In both KD groups, changes in the levels of PA/PAI-1 complex showed patterns similar to those of PAI-1 levels. There was no difference in PA/PAI-1 complex levels between the $2 \mathrm{KD}$ groups at any time during the disease course. Thus, in group 2, the ratio of PA/PAI-1 complex to PAI-1 remained low and was significantly lower than that of group 1 after IVGG, suggesting persistent fibrinolytic imbalance after IVGG in KD patients with CALs.

To further elucidate the pathologic significance of the PAPAI-1 system in CAL development, we compared individual changes in PAI-1 and the ratio of PA/PAI-1 complex to PAI-1 between group 2 patients and group 1 patients whose PAI-1 levels before IVGG were higher than the lowest value of group
$2(80 \mathrm{ng} / \mathrm{mL} ; 9$ patients) or whose ratio of PA/PAI-1 complex to PAI-1 before IVGG was lower than the highest value of group $2(0.21 ; 15$ patients). Mean values of PAI- 1 and ratio of PA/PAI-1 complex to PAI-1 for these group 1 patients were similar to those of group 2 patients (PAI-1, $138 \pm 14 \mathrm{ng} / \mathrm{mL}$; ratio of PA/PAI-1 complex to PAI- $1,0.11 \pm 0.02 ; p=\mathrm{NS}$ versus group 2). As shown in Fig. 3, PAI-1 levels of these group 1 patients significantly decreased after IVGG $(85 \pm 18$ $\mathrm{ng} / \mathrm{mL}, p<0.01$ versus before IVGG), whereas those of group 2 patients remained unchanged. Thus, PAI- 1 after IVGG was significantly higher for group 2 patients than for this subset of group 1 patients $(p<0.01)$. A PAI- 1 level of $80 \mathrm{ng} / \mathrm{mL}$ or greater, both before and after IVGG, had a sensitivity of $100 \%$ and specificity of $70 \%$ for prediction of development of CALs. Similarly, the ratio of PA/PAI-1 complex to PAI-1 markedly increased after IVGG in these group 1 patients $(0.23 \pm 0.02, p$ $<0.01$ versus before IVGG), but not in group 2, indicating a 
PAI-1

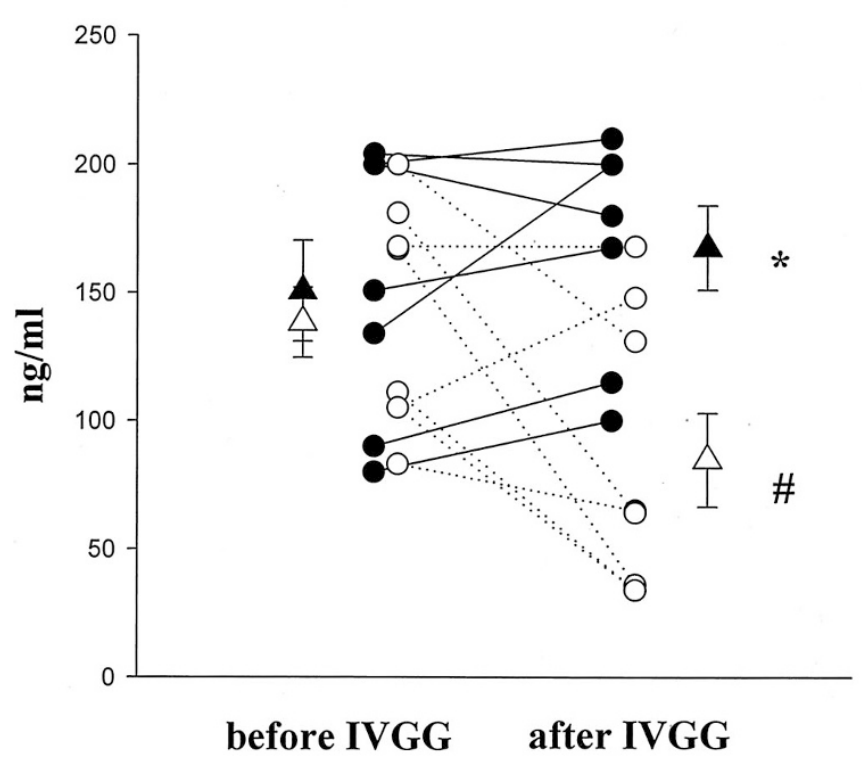

PA/PAI-1 Complex vs. PAI-1 Ratio

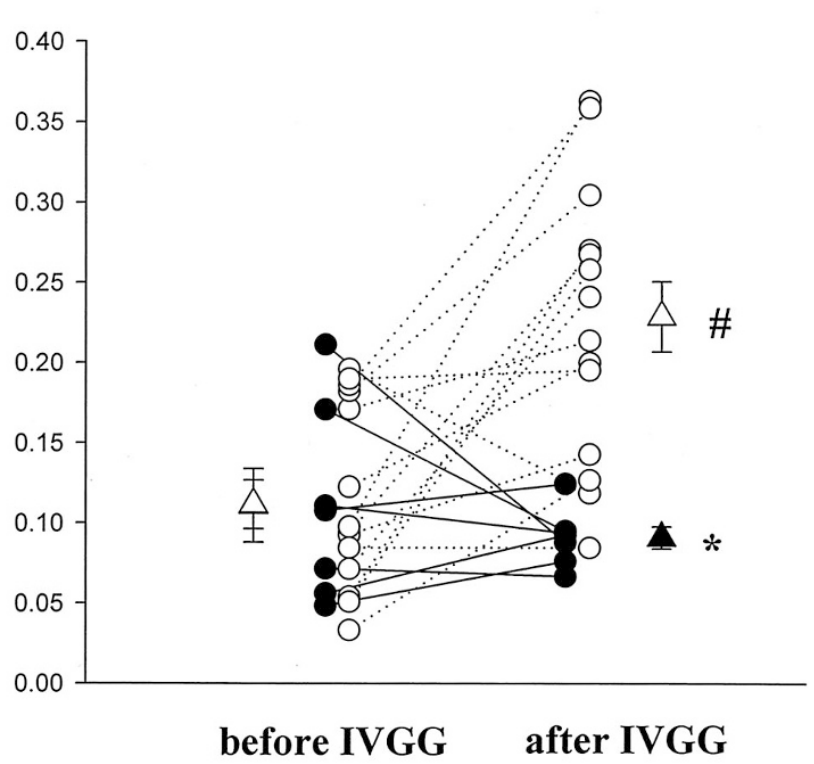

Figure 3. Individual changes in PAI-1 and the ratio of PA/PAI-1 complex to PAI-1 in selected group 1 patients (open circles) and group 2 patients (closed circles), before and after i.v. gammaglobulin therapy. Mean changes are indicated by triangles. $\bigcirc$, group 1; $\bullet$, group $2 ; \triangle$, group 1 mean; $\mathbf{\Delta}$, group 2 mean; $* p<0.05 v s$. group $1 ; \# p<0.05$ vs. before IVGG.

significantly higher ratio of $\mathrm{PA} / \mathrm{PAI}-1$ complex to $\mathrm{PAI}-1$ in these group 1 patients than in group $2(p<0.01)$. Thus, persistent fibrinolytic imbalance that favors inhibition of the PA/plasmin pathway appears to be closely related to CAL development in KD.

\section{DISCUSSION}

The present results demonstrate that circulating levels of PAI-1 and PA/PAI-1 complex are markedly elevated in KD. More importantly, PAI-1 levels of KD patients with CALs were significantly higher than those of KD patients without CALs before IVGG and remained elevated after IVGG, with imbalance against PA/PAI-1 complex levels. These data suggest that circulating levels of PAI-1 and the ratio of PA/PAI-1 complex to PAI-1 are related to the severity of vascular damage during the acute phase of $\mathrm{KD}$, and that $\mathrm{KD}$ patients with high PAI-1 levels (both absolute and relative to PA/PAI-1 complex) are susceptible to CALs.

The physiologic condition of extracellular matrix is maintained by a rigorously controlled balance between the activity of proteases of its component proteins and the activity of endogenous inhibitors of those proteases. MMPs and their inhibitors, known as tissue inhibitors of MMPs (TIMPs), play central roles in this process (3). Accelerated matrix breakdown caused by increased activity of MMPs and/or quantitative imbalance between MMPs and TIMPs is involved in several pathologic conditions, including rheumatoid arthritis (17), tumor metastasis (18), and heart failure $(19,20)$. There is also increasing evidence that MMP levels are increased in arterial walls of abdominal aortic aneurysms, and that this increase contributes to wall degradation and aneurysm formation (4-6, 21, 22).

PAs convert plasminogen into plasmin. PAI-1 is the primary physiologic inhibitor of plasminogen activation (23). Because most MMPs are secreted as zymogens, MMP activity in the extracellular space is dependent on zymogen activation (3). In vitro studies suggest that the PA/plasmin system is a major pathway of MMP activation $(7,8)$. For example, plasmin can convert proMMP-3 into active MMP-3 $(24,25)$, which in turn activates MMP-1 and MMP-9 (25, 26). PA/plasmin also directly activates MMP-9, -12 and $-13(7,8,27)$. There have also been in vivo studies indicating that the PA/plasmin system plays a predominant role in regulating MMP activity and MMPinduced pathologic matrix degradation. In a mouse model of acute myocardial infarction, blocking plasminogen activation by temporal infusion of PAI-1 prevented rupture of ventricular aneurysm (28), in which ventricular wall destruction by increased MMP activity is thought to be a main mechanism (29). It has also been shown that adenoviral transfer of the PAI-1 gene reduces tumor metastasis (30); in metastasis, extracellular matrix lysis is necessary for movement of transformed cells in tissues. In addition, Allaire et al. have reported that, in a rat model of aortic aneurysm, blockade of PAs by local overexpression of PAI-1 prevents formation of aneurysms and arterial rupture by inhibiting MMP activation (9). The above findings indicate that the PA/Plasmin system can trigger extracellular matrix degradation by activating the MMP pathway of proteolysis, resulting in the pathologic conditions mentioned above. 
Interestingly, PAI-1 levels have also been found to be elevated in patients with increased activity of PA/plasmin and MMPs $(31,32)$. Despite its presence in lesions where pathologic extracellular matrix lysis occurs, the concentration of PAI-1 may be inadequate for full blockade of the PA-MMP system and related lesion development.

A situation similar to that described above may be the increased PAI-1 levels in KD patients with CALs. In a previous study, we showed that MMPs may also be intimately involved in coronary arterial wall destruction and the resultant formation of coronary aneurysms in KD (12). Given the reported inhibitory role of PAI-1 in MMP activation, the present results, which indicate increased levels of PAI-1 in KD with CALs, suggest that PAI-1 levels increase in response to coronary arterial wall injury, and that PAI-1 helps to protect against arterial wall destruction and resultant CAL formation, although the protective effects of PAI-1 are insufficient in KD. This may be analogous to a situation observed in heart failure, where atrial and brain natriuretic peptides that are cardioprotective are elevated in proportion to the severity of heart failure but are not sufficient to prevent heart failure progression (33).

It is also important to note that, in addition to its inhibitory action against MMP activation, PAI-1 plays diverse pathophysiologic roles in the process of tissue remodeling. Several previous studies using animal models of arterial injury have indicated that PAI-1 transiently impairs cell migration and thereby limits neointimal formation early after injury (34-36). However, PAI-1 subsequently promotes fibrin accumulation and cell proliferation, resulting in neointimal growth at later time points (34). Varying effects of PAI-1 during the time course of tissue remodeling have also been suggested by Heymans et al. who used a rat model of acute myocardial infarction (28). They reported that urokinase-type PA deficiency completely protected against cardiac rupture early after myocardial infarction, but those mice consequently died of cardiac failure due to impaired scar formation and infarct revascularization. Only temporary administration of PAI-1 or MMP inhibitor completely protected mice against rupture without aborting infarct healing (28). Therefore, although increased levels of PAI-1 in the early stage of KD may protect against vascular wall destruction, prolonged activation of PAI-1 at a later stage may produce undesirable effects by promoting cell proliferation and fibrosis. Furthermore, in plasma, PAI-1 is a critical determinant of endogenous thrombolytic activity (23). At later stages of $\mathrm{KD}$, when platelet counts are markedly elevated, increased PAI-1 may also increase the risk of thrombotic events, particularly if CALs have already developed.

The various possible actions of PAI-1 at different stages of tissue remodeling may also have important implications in therapeutic strategy for preventing CALs in KD. Although our results suggest that blocking the $\mathrm{PA} /$ plasmin pathway is a viable therapeutic approach to preventing KD-related CALs, such therapeutic intervention may have to be limited to the very early stage of illness.

Because we do not have conclusive evidence that PAI-1 has inhibitory effects on MMP activities in KD, we must also consider the possibility that higher PAI-1 levels in patients with CAL than in those without CAL are due to a causative role of PAI-1 in development of CAL. Therefore, further clinical studies of effects of PA inhibition on MMP activities and resultant coronary outcome are needed to define the roles of PAI-1 in CAL formation in KD. Data from such studies may support the use of $\mathrm{PA} /$ plasmin inhibitors for prevention of coronary artery complications in patients with KD.

The results of the present study indicate that KD patients with persistently elevated PAI-1 with imbalance against PA levels are highly susceptible to CAL development. The individual changes in PAI-1 shown in Fig. 3 strongly support this conclusion. Thus, circulating levels of PAI-1 and the ratio of PA/PAI-1 complex to PAI-1 may be useful as predictive markers for CAL development in KD. There is controversy over whether high-dose gammaglobulin therapy should be applied to all KD patients (37); consequently, the decision of whether to use gammaglobulin is often based on one of several risk stratification scores for development of CAL (38). PAI-1 level may provide additional information about the risk of CAL development, thus helping determine whether to use gammaglobulin. In addition, although high-dose IVGG therapy can reduce the risk of development of CAL, some patients who receive this treatment still develop CAL. Additional therapy, such as corticosteroids (39) or repeated administration of IVGG (40), may be required to further reduce the incidence of CAL development. The present results suggest that such additional therapy should be considered for KD patients with elevated PAI-1 that persists after IVGG therapy.

It is well known that levels of various cytokines, such as tumor necrosis factor (TNF)- $\alpha$, interleukin (IL)-1 $\beta$ and IL-6, are elevated in KD patients $(41,42)$. Recent studies by Seki et $a l$. indicate that IL-1 $\beta$ plays a key role in inducing PAI-1 expression in response to local tissue injury $(43,44)$. Interestingly, Leung et al. reported that high levels of IL-1 were secreted from mononuclear cells in Kawasaki disease patients, and that IL-1 secretion remained high after gammaglobulin therapy in patients with CAL but fell to normal levels in patients without CAL (45). This is very similar to the pattern of PAI-1 expression observed in the present study; it suggests that there is a close link between IL-1 and PAI-1 in KD, and that both proteins have pathophysiological significance in $\mathrm{KD}$. In addition, reports by Seki et al. suggest that TNF- $\alpha$ is important for the induction of PAI-1 (43). The above findings indicate the need for studies that directly examine relationships between these cytokines and PAI-1 in $\mathrm{KD}$, and their contributions to development of CAL.

In summary, we found that circulating levels of PAI-1 are markedly elevated in KD patients and are closely related to CAL formation. The present data suggest that circulating levels of PAI-1 are useful as a predictive marker for CAL development, and that they can serve as a therapeutic target for prevention of CALs. Prospective studies could help to clarify the usefulness of these potential applications.

Acknowledgments. The authors wish to thank Drs. T. Nakamura, Y. Ogawa (Saitama Medical Center), R. Tochigi (Kumagaya General Hospital), and M. Ogata (Ogawa Red Cross Hospital) for their valuable assistance in data collection. 


\section{REFERENCES}

1. MacSweeney ST, Powell JM, Greenhalgh RM 1994 Pathogenesis of abdominal aortic aneurysm. Br J Surg 81:935-941

2. Anidjar S, Kieffer E 1992 Pathogenesis of acquired aneurysms of the abdominal aorta. Ann Vasc Surg 6:298-305

3. Parks WC, Mecham RP 1998 Matrix Metalloproteinases. Academic Press, San Diego, pp 1-21

4. Thompson RW, Holmes DR, Mertens RA, Liao S, Botney MD, Mecham RP, Welgus HG, Parks WC 1995 Production and localization of 92-KD gelatinase in abdominal aortic aneurysms: an elastolytic metalloproteinase expressed by aneurysm-infiltrating macrophages. J Clin Invest 96:318-326

5. Davies MJ 1998 Aortic aneurysm formation. Lessons from human studies and experimental models. Circulation 98:193-956

6. Tamarina NA, McMillan WD, Shively VP 1997 Expression of matrix metalloproteinases and their inhibitors in aneurysms and normal aorta. Surgery 1222:264-271

7. Baramova EN, Bajou K, Remacle JM, L'Hoir C, Krell HW, Weidle UH, Noel A, Foidart JM 1997 Involvement of PA/plasmin system in the processing of pro-MMP-9 and in the second step of pro-MMP-2 activation. FEBS Lett 405:157-162

8. Mazzieri R, Masiero L, Zanetta L, Monea S, Onisto M, Garbisa S, Mignatti P 1997 Control of type IV collagenase activity by components of the urokinase-plasmin system: a regulatory mechanism with cell-bound reactants. EMBO J 16:2319-2332

9. Allaire E, Hasenstab D, Kenagy RD 1998 Prevention of aneurysm development and rupture by local overexpression of plasminogen activator inhibitor-1. Circulation 98:249-255

10. Dajani AS, Taubert KA, Gerber MA, Shulman ST, Ferrieri P, Freed MD 1993 Diagnosis and therapy of Kawasaki disease. Circulation 87:1776-1780

11. Senzaki H, Suda M, Noma S, Kawaguchi H, Sakakihara Y, Hishi T 1994 Acute heart failure and acute renal failure in Kawasaki disease. Acta Paediatr Jpn 36:443-447

12. Senzaki. H, Masutani S, Kobayashi J, Kobayashi T, Nakano H, Nagasaka H, Sasaki N, Asano H, Kyo S, Yokote Y 2001 Circulating Matrix Metalloproteinases and Their Inhibitors in Patients with Kawasaki Disease Circulation 104:860-863

13. Sakata Y, Murakami T, Noro A, Mori K, Matsuda M 1991 The specific activity of plasminogen activator inhibitor-1 in disseminated intravascular coagulation with acute promyelocytic leukemia. Blood 77:1949-1957

14. Chandler WL, Alessi MC, Aillaud MF, Henderson P, Vague P, Vague IJ 1997 Clearance of tissue plasminogen activator (TPA) and TPA/plasminogen activator inhibitor type 1 (PAI-1) complex. Relationship to elevated TPA antigen in patients with high PAI-1 activity levels. Circulation 96:761-768

15. Japan Kawasaki disease research committee 1984 Diagnostic guidelines of Kawasak disease, 4th Rev Ed. Japan Kawasaki disease research committee, Tokyo

16. Brown NJ, Agirbasli MA, Williams GH, Litchfield R, Vaughan DE 1998 Effect of activation and inhibition of the rennin-angiotensin system on plasma PAI-1. Hypertension 32:965-971

17. Woessner JF 1991 Matrix metalloproteinases and their inhibitors in connective tissue remodeling. FASEB J 5:2145-2154

18. Strtler-Stevenson WG 1996 Dynamics of matrix turnover during pathologic remodeling of the extracellular matrix. Am J Pathol 148:1345-1350

19. Senzaki H, Gluzband YA, Pak PH, Crow MT, Janicki JS, Kass DA 1998 Synergistic exacerbation of diastolic stiffness from short-term tachycardia-induced cardiodepression and angiotensin II. Circ Res 82:503-512

20. Senzaki H, Gluzband A, Crow MT, Kass DA $2000 \beta$-adrenergic blockade prevents MMP activation and diastolic dysfunction in short-term tachycardia-induced cardiodepression with angiotensin II. Circ Res 86:807-815

21. Vine N, Powell JT 1991 Metalloproteinases in degenerative aortic disease. Clin Sci 816:233-239

22. Carrell TWG, Burnand KG, Wells GMA, Clements JM, Smith A 2002 Stromelysin-1 (matrix metalloproteinase-3) and tissue inhibitor of matrix mettaloproteinase-3 are overexpressed in the wall of abdominal aortic aneurysms Circulation 105:477-482

23. Loskutoff DJ, Sawdey M, Mimuro J 1989 Type 1 plasminogen activator inhibitor. Prog Hemost Thromb 9:87-115
24. Nagase H, Enghild JI, Suzuki K, Salvesen G 1990 Stepwise activation mechanisms of the precursor of matrix metalloproteinase 3 (stromelysin) by proteases and (4aminophenyl)mercuric acetate. Biochemistry 29:2783-5789

25. He CS, Wilhelm SM, Pentland AP, Marmer BL, Grant GA, Eisen AZ, Goldberg GI 1989 Tissue cooperation in a proteolytic cascade activating human interstitial collagenase. Proc Natl Acad Sci USA 86:2632-2636

26. Shapiro SD, Fliazar CJ, Broekelmann TJ, Mecham RP, Senior RM, Welgus HG 1995 Activation of the $92-\mathrm{kDa}$ gelatinase by stromelysin and 4-aminophenylmercric acetate: differential processing and stabilization of the carboxyl-terminal domain by tissue inhibitor of metalloproteinases (TIMP). J Biol Chem 270:6351-6356

27. Carmeliet P, Moons L, Lijnen R, Baes M, Lemaitre V, Tipping P, Drew A, Eeckhout Y, Shapiro S, Lupu F, Collen D 1997 Urokinase-generated plasmin activates matrix metalloproteinases during aneurysm formation. Nat Genet 17:439-444

28. Heymans S, Luttun A, Nuyens D, Theilmeier G, Greenmers E, Moons C, Dyspersin GD, Cleutjens JPM, Shipley M, Angellilo A, Levi M, Nube O, Baker A, Keshet E, Lupu F, Herbert JM, Smits JFM, Shapiro SD, Baes M, Borgers M, Collen D, Daemen MJAP, Carmeliet P 1999 Inhibition of plasminogen activators or matrix metalloproteinases prevents cardiac rupture but impairs therapeutic angiogenesis and causes cardiac failure. Nat Med 5:1135-1142

29. Rohde LE, Ducharme A, Arroyo LH, Aikawa M, Sukhova GH, Lopez-Anaya A, McClure KF, Mitchell PG, Libby P, Lee RT 1999 Matrix metalloproteinase inhibition attenuates early left ventricular enlargement after experimental myocardial infarction in mice. Circulation 99:3063-3070

30. Praus M, Wauterickx K, Collen D, Gerard RD 1999 Reduction of tumor cell migration and metastasis by adenoviral gene transfer of plasminogen activator inhibitors. Gene Ther 6:227-236

31. Simpson AJ, Booth NA, Moore NR, Bennett B 1991 Distribution of plasminogen activator (PAI-1) in tissues. J Clin Pathol 44:139-143

32. Kikuchi H, Shimada W, Nonaka T, Ueshima S, Tanaka S 1996 Significance of serine proteinase and matrix metalloproteinase systems in the destruction of human articular cartilage. Clin Exp Pharmacol Physiol 23:885-889

33. Mcmurray J, Pfeffer MA 2002 New therapeutic options in congestive heart failure: part I. Circulation 105:2099-2106

34. Deyoung MB, Tom C, Dichek DA 2001 Plasminogen activator inhibitor type 1 increases neointimal formation in balloon-injured rat carotid arteries. Circulation 104:1972-1977

35. Carmeliet P, Moons L, Lijnen R, Janssens S, Lupu F, Collen D, Gerard RD 1997 Inhibitory role of plasminogen activator inhibitor-1 in arterial wound healing and neointimal formation: a gene targeting and gene transfer study in mice. Circulation 96:3180-3191

36. Hasenstab D, Lea H, Clowes AW 2000 Local plasminogen activator inhibitor type 1 overexpression in rat carotid artery enhances thrombosis and endothelial regeneration while inhibiting intimal thickening. Arterioscler Thromb Vasc Biol 20:853-859

37. Gersony WM 1998 Predicting coronary artery aneurysms in Kawasaki disease. Am J Cardiol 81:1162-1164

38. Harada K 1991 Intravenous -globulin treatment in Kawasaki disease. Acta Paediatr Jpn 33:805-810

39. Shinohara M, Sone K, Tomomasa T, Morikawa A 1999 Corticosteroids in the treatment of the acute phase of Kawasaki disease. J Pediatr 135:465-469

40. Wallace CA, French JW, Kahn SJ, Sherry DD 2000 Initial intravenous gammaglobulin treatment failure in Kawasaki disease. Pediatrics 105:E78

41. Lang BA, Silverman ED, Laxer RM 1989 Spontaneous tumor necrosis factor production in Kawasaki disease. J Pediatr 115:939-943

42. Barron K 1991 Immune abnormalities in Kawasaki disease: prognostic implications and insight into pathogenesis. Cardiol Young 1:206-211

43. Seki T, Healy AM, Fletcher DS, Noguchi T, Gelehrter TD 1999 IL-1 $\beta$ mediates induction of hepatic type 1 plasminogen activator in response to local tissue injury. Am J Physiol 277(Gastrointest Liver Physiol 40):G801-G809

44. Seki T, Gelehrter TD 1996 Interleukin-1 induction of type-1 plasminogen activator inhibitor (PAI-1) gene expression in the mouse hepatocyte line, AML 12. J Cell Physiol 168:648-656

45. Leung DY, Cotran RS, Kurt-Jones E, Burns JC, Newburger JW, Pober JS 1989 Endothelial cell activation and high interleukin-1 secretion in the pathogenesis of acute Kawasaki disease. Lancet 2(8675):1298-1302 